\title{
Early-stage studies to larger-scale trials: investigators' perspectives on scaling-up childhood obesity interventions
}

\author{
L. von Klinggraeff ${ }^{*}$, R. Dugger ${ }^{1}$, A. D. Okely², D. Lubans³ , R. Jago ${ }^{4}$, S. Burkart, R. G. Weaver, B. Armstrong, \\ C. D. Pfledderer ${ }^{1}$ and M. W. Beets ${ }^{1}$
}

\begin{abstract}
Background: Pilot/feasibility studies play an important role in the development and refinement of behavioral interventions by providing information about feasibility, acceptability, and potential efficacy. Despite their importance and wide-spread use, the approaches taken by behavioral scientists to scale-up early-stage studies to larger-scale trials has received little attention. The aim of our study was to understand the role that pilot studies play in the development and execution of larger-scale trials.
\end{abstract}

Methods: We conducted interviews with childhood obesity researchers who had published pilot behavioral interventions and larger-scale trials of the same or similar interventions. Questions were asked about the role of pilot studies in developing larger-scale trials and the challenges encountered when scaling-up an intervention based upon pilot findings. Data were coded and analyzed using an inductive analytic approach to identify themes.

Results: Twenty-four interventionists (54\% women, 37-70 years old, mean 20 years since terminal degree) completed a total of 148 pilot studies across their careers (mean 6.4, range 1-20), of which 59\% were scaled-up. Scaling was described as resource intensive and pilot work was considered essential to successfully competing for funding by $63 \%$ of the sample $(n=15)$. When asked to define a high-quality pilot study, interventionists described studies that allowed them to evaluate two independent factors: components of their intervention (e.g., acceptability, feasibility) and study parameters (e.g., sample size, measures). Interventionists expressed that more process implementation measures, different study designs, and additional iterations could improve decisions to scale-up. Most agreed that pilot studies were likely to produce inflated estimates of potential efficacy though only nine interventionists provided potential solutions for decreasing inflated measures of efficacy. Suggested major causes of inflated effects included high levels of oversight in pilot studies (e.g., researcher support), reliance on subjective measures, and utilizing convenience or highly motivated samples. Potential solutions included designing pilots for real-world implementation, only conducting randomized controlled pilot studies, and pre-registering pilot studies.

Conclusions: Pilot studies purposes are multifaceted and deemed essential to obtaining funding for larger-scale trials. Clarifying the form and function of preliminary, early-stage research may enhance the productive utilization of early-stage studies and reduced drops in efficacy when transitioning to larger scale studies.

\footnotetext{
*Correspondence: vonklinl@email.sc.edu

${ }^{1}$ Arnold School of Public Health, University of South Carolina, 921

Assembly Street, Room 130, Columbia, SC 29205, USA

Full list of author information is available at the end of the article
} otherwise in a credit line to the material. If material is not included in the article's Creative Commons licence and your intended use is not permitted by statutory regulation or exceeds the permitted use, you will need to obtain permission directly from the copyright holder. To view a copy of this licence, visit http://creativecommons.org/licenses/by/4.0/. The Creative Commons Public Domain Dedication waiver (http://creativecommons.org/publicdomain/zero/1.0/) applies to the data made available in this article, unless otherwise stated in a credit line to the data. 
Keywords: Childhood obesity, Scalability, Intervention, Translation, Pilot

\section{Background}

To achieve widespread improvements in non-communicable diseases rates, interventions shown to be efficacious in smaller-scale studies need to be effective when "scaledup" for widespread impact [1-5]. Successful implementation of behavioral interventions at-scale is a matter of international importance [6-10], though successful execution of effective scaling in behavioral health is difficult [11]. Recent reviews have revealed a frequent pattern whereby scaled-up interventions that have assessed for effectiveness at the pilot/feasibility stage often produce non-significant and/or substantially smaller effects compared to earlier pilot/feasibility trials $[1,12-14]$. This may be due in part to a repeated pattern of "failure to scale", "voltage drop", or "scale-up penalty." These factors have been consistently documented, indicating a need for increased attention to the early stage of intervention development and testing. Failure of behavioral interventions to effectively scale-up wastes valuable resources and significantly delays progress in reducing non-communicable diseases. This phenomenon represents a critical barrier to progress in developing effective behavioral interventions that perform once scaled-up.

Though the terminology and purpose of preliminary early-stage studies, commonly referred to as pilot or feasibility studies is debated [15-17], they are generally acknowledged as a fundamental and critical step in the process of intervention development $[18,19]$. Researchers use pilot studies to prepare for a larger-scale iteration of a same or similar intervention by providing conceptual clarity (i.e., "proof of concept"), "optimization" of complex interventions [20], provide experience navigating potential obstacles likely to occur in intervention implementation. They also provide information about perception among participants' that the treatment is agreeable (i.e., acceptability), intervention feasibility, evaluation feasibility, and preliminary efficacy $[17,21]$. Among the many models proposed for scaling-up health interventions [19], such as the Obesity Related Behavioral Intervention Trials (ORBIT) model [18] or the NIH Stage Based Model for Behavioral Intervention Development [22] nearly all frame the implementation process as an cumulative, multi-phased process beginning with formative or pilot-related work.

How behavioral scientists actually approach intervention development from early-stage studies to larger-scale trials remains unexplored in the literature. Understanding the experiences of senior researchers who conduct pilot studies and subsequent larger trials could help elucidate the purpose, motivations, and difficulties of piloting behavioral interventions and add depth and contextual significance to our understanding of the scaling processes. A clear understanding of the purpose and function of pilot studies can help the field adopt and integrate improved practices in pilot study execution [23]. The purpose of our study was to conduct qualitative interviews with lead authors of a published pilot study that had a subsequent published larger-scale trial on a topic related to childhood obesity in order to better understand to understand, the role that pilot studies play in the development and execution of larger-scale trials.

\section{Methods}

\section{Interview guide development}

We developed a semi-structured interview guide using a collaborative process. Questions were aimed at eliciting participants' personal experiences in the design and scaling of behavioral interventions and were based on the research team's experiences as interventionists as well as theories of program implementation [24]. Questions were initially developed and reviewed by a team of content experts (RJ, DL, AO) who evaluated them in terms of clarity, content, and alignment with the research question.

A preliminary interview was conducted to ascertain the length, pace, and the suitability of question sequence for the interviews. For the preliminary interview, one individual was selected from the research team who had also provided input in question development (DL). Based on feedback from the preliminary interview, questions were amended for additional clarity and a semi-structured format was chosen to facilitate a conversational interviewing style, allowing the interviewer to follow-up with additional questions as needed. The finalized formal interview guide consisted of 10 predetermined openended questions.

\section{Recruitment}

Thirty-eight previously identified authors of published pilot/feasibility studies and subsequent larger-scale trials of behavioral interventions targeting childhood obesity were eligible for participation. Procedures used to identify all possible qualifying pilot studies and their subsequent well powered trials have been described elsewhere [12].

All 38 authors were invited to participate in the study by email in November of 2019. The initial recruitment email introduced the study and the study's purpose. Each 
email provided potential participants with citations for the specific pilot/feasibility and larger-scale studies that had led them to be included in the study. Emails included definitions for "pilot/feasibility study" and "behavioral intervention" to help orient participants. Participants were encouraged to respond to the email and select one of three listed time/dates for an interview lasting approximately 30 to $60 \mathrm{~min}$ in length. After the initial email, non-responding participants were contacted up to three more times to participate. After no response from the third contact, no more attempts to recruit participants were made.

All procedures were approved by the first author's institutional review board (registration number Pro00086876) prior to enrollment of the first participant and were consistent with the ethical standards outlined by the Helsinki Declaration of 1975, as revised in 2000 [25].

\section{Data collection Interviews}

Interviews took place through video conference software (Zoom Video Communications, San Jose, CA) for participants living outside of the USA and/or via phone for U.S. residents. All interviews were conducted by one of the authors $(\mathrm{MB})$. During the interview, the interviewer guided the conversation through the predetermined questions attempting to not make any leading statements. Questions were followed up with probing questions for clarification and to explore new ideas as necessary. All interviews were audio recorded and notes were taken throughout the interview to capture initial thoughts and observations. Participants were read an IRB-approved script which informed them of the interview's purpose and the use of the data. Participants provided verbal consent to have their interview recorded and used for the study.

\section{Transcription}

All transcription was done verbatim using Otter.ai (AISense, Los Altos, CA) and verified by trained research assistants. Transcriptions were uploaded into NVivo 12 Plus (QSR International, Doncaster Australia) for coding and synthesis.

\section{Analysis}

Two trained research assistants (LV, RD) coded, analyzed, and synthesized the data using analytic induction $[26,27]$. Both research assistants had completed formal coursework in qualitative methodology for public health research and had assisted in at least two prior, qualitative studies both as interviewers and coders.
Modified analytic induction was appropriate for this study, given that we expected interventionists who are interviewed to explain successful/unsuccessful translation of pilot to large-scale intervention results by citing factors that can be traced to both well-documented mediating variables in implementation science in addition to novel, less documented experiences expressed by participants.

This process was comprised of four key steps: data immersion, creating codes, arranging themes, and formulating and refine the explanatory hypothesis. The first step was immersion which allowed for a detailed examination of the data during which emergent themes began to surface. Once the data had been examined, the two independent coders read the transcripts in batches of 2-3 transcripts and compared coding line by line, discussing and resolving disagreements. Themes were identified a priori, and emergent themes identified during data emersion were incorporated. Themes were identified in the literature as prevalent features of scaled-up interventions [12]. Coders met weekly to review themes and discussed any disagreements until consensus was reached. The final step was to formulate and refine the explanatory hypothesis. This was done using an arrangement of codes to identify common dimensions across themes [28]. Once a preliminary explanatory hypothesis was reached, transcripts were revisited, and hypotheses redefined and revised as negative cases were detected. During the arrangement of themes and the formation of the explanatory hypotheses, the two coders debriefed with the principal investigator (MB) every other week. Debriefs included reviewing data organization, debating thematic arrangement and revisiting points of disagreement between the two coders.

\section{Trustworthiness}

Guided by Shenton's provisions to ensure credibility, transferability, dependability, and confirmability in qualitative research [29], intentional and systematic methodological steps were embedded within the study to improve trustworthiness of the data collection, analysis, and synthesis. To support the credibility of the findings, consistent, scheduled peerengagement occurred between authors during every phase of the project development and execution. This included question development (peer-scrutiny; $\mathrm{MB}$, $\mathrm{AO}, \mathrm{DL}, \mathrm{RJ}$ ), data collection (reflective commentary; $\mathrm{MB}, \mathrm{RW}$ ), and data analysis (peer-debriefing; RD, LV, MB). Our study was informed by a meta-epidemiologic study on the same population of behavioral researchers as well as work that has consistently 
documented the "failure to scale" phenomena [12]. This conceptual foundation supports the transferability of the findings and allows for allows for parallels to be drawn between the quantitative and qualitative data. This approach of using quantitative data as a foundation for qualitative exploration, also serves to bolster the confirmability of the findings, as does the detailed description of the analytic processes employed during data synthesis [29].

\section{Results}

Of the 38 individuals eligible to participate, a total of 24 interviews were completed (62\%). The remaining eligible participants did not respond to the email invitations. Interviews varied in length ranging from 32 to $68 \mathrm{~min}$ (mean 47.45). The interviews were conducted between November 2019 and March 2020. Participants predominantly self-identified as White (92\%), were employed by universities (92\%) and currently or formally held tenured positions (87\%) with an average of 20 years of research experience (range 11-35 years). The average age of participants was 50.5 years (SD 8.8) and $46 \%$ were male. Most participants resided in Australia (35\%), the USA (38\%), and the UK (19\%).

Through modified analytic induction 20 themes were generated containing 111 subthemes which were organized into four broad categories, or stages: (1) conceptualization of an intervention, (2) interpretation of results, (3) scaling, and (4) reflection (Table 1).

\section{Category 1: conceptualization of an intervention}

Across the interviews, pilot studies were discussed as playing a valuable role in molding concepts and approaches into an intervention that could be scaledup. This involved critically examining and solidifying ideas into processes or protocols that could be executed, as well as choosing target outcomes, participant populations, and measurement tools. The choice to conduct early intervention development work was driven by a desire to establish a convincing case for further resource investments in a later, larger-scale trial. Investigators hoped to provide indications that the investigators could execute the logistics of the trial (e.g., recruit participants, deliver intervention, measure outcomes), while simultaneously providing evidence that the intervention has potential impact on either primary or secondary (i.e., intermediary) outcomes, referred to as a preliminary signal. The following elements were identified by interviewees as metrics that would provide valuable information for later interpretation when designing a pilot study, prior to scaling-up an intervention.

\section{Gathering information}

In the initial design of the interventions, researchers sought multiple sources of information to inform intervention components. These included reviews of the literature and input from key stakeholders to inform specific intervention content (quote 1a). Emphasis was placed on generating new or novel ideas for testing, the integration of existing evidence-based practices, and targeting the mechanisms of behavior change (quote $1 \mathrm{~b}$ ).

\section{Measurement logistics}

Pilot studies were described as an opportunity to determine whether study measures would result in sufficient amounts of quality data. For instance, investigators described wanting to gauge whether participants would tolerate particular measures, such as dual X-ray absorptiometry, return rates of measurement tools (e.g., accelerometers), complete self-report questionnaires, determine if measures were age appropriate, and/or refine the content/focus of certain measures (e.g., survey redesign; quote 2a). Study specific contexts drove this component of the pilot study, such as delivering mHealth interventions where measures would be completed without direct contact with research staff (quote $2 b$ ).

\section{Trial parameters}

A key indicator for moving forward with the scale-up of an intervention was positive results on trial-related parameters. Trial-related parameters were described as target population recruitment and whether they could be recruited in sufficient numbers, retention of participants over the duration of the intervention (i.e., low attrition rates) and participant engagement in the interventionrelated activities (e.g., attend the number of prescribed sessions) to receive an adequate dose for an effect. Receptivity of being randomized to conditions was also considered (quote 3a). Other trial-related parameters involved process implementation measures such feasibility and fidelity that were embedded within pilot studies, along with measures of acceptability and satisfaction (quote $3 \mathrm{~b}$ ) from both participants, and where appropriate, delivery agents.

\section{Mirror larger trial}

Making an intentional effort to design pilot studies a priori as if they were smaller versions of the anticipated larger, future trial was consistently mentioned. Several investigators were adamant that trial and intervention protocols be as close as possible to the future larger-scale, more well-powered trial $(n=3$; quote $4 \mathrm{a})$ while others placed emphasis on aligning conceptual components 


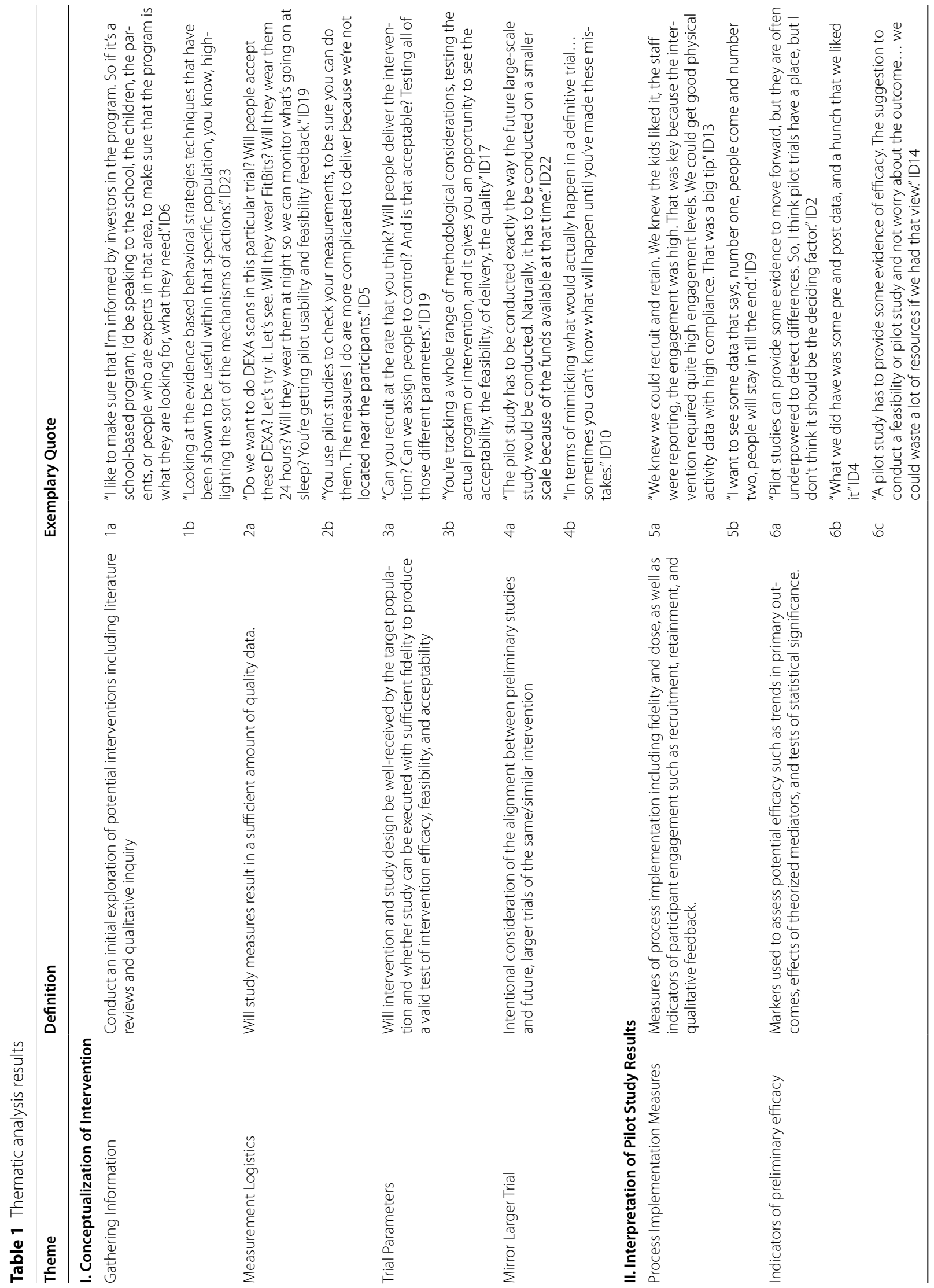




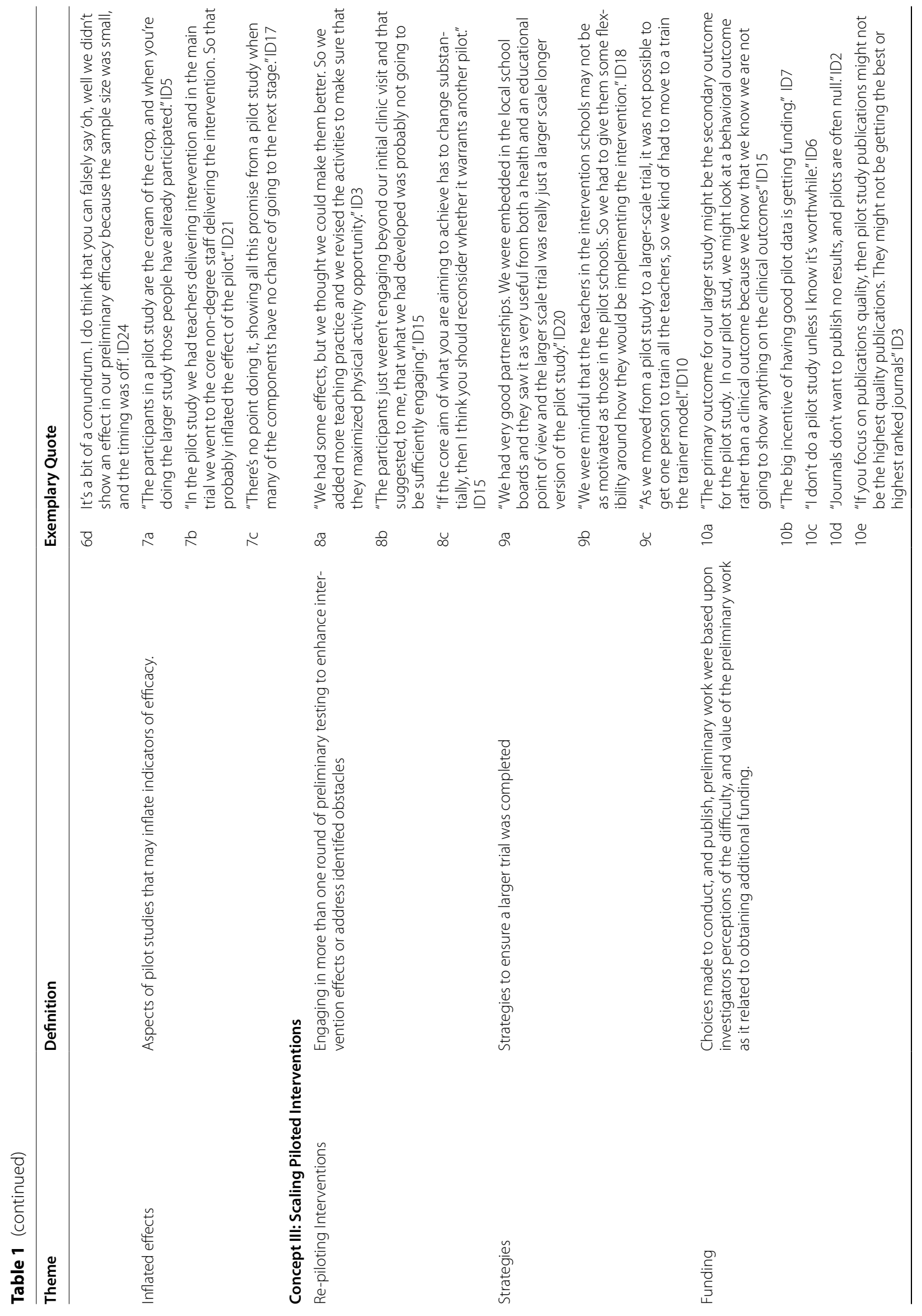




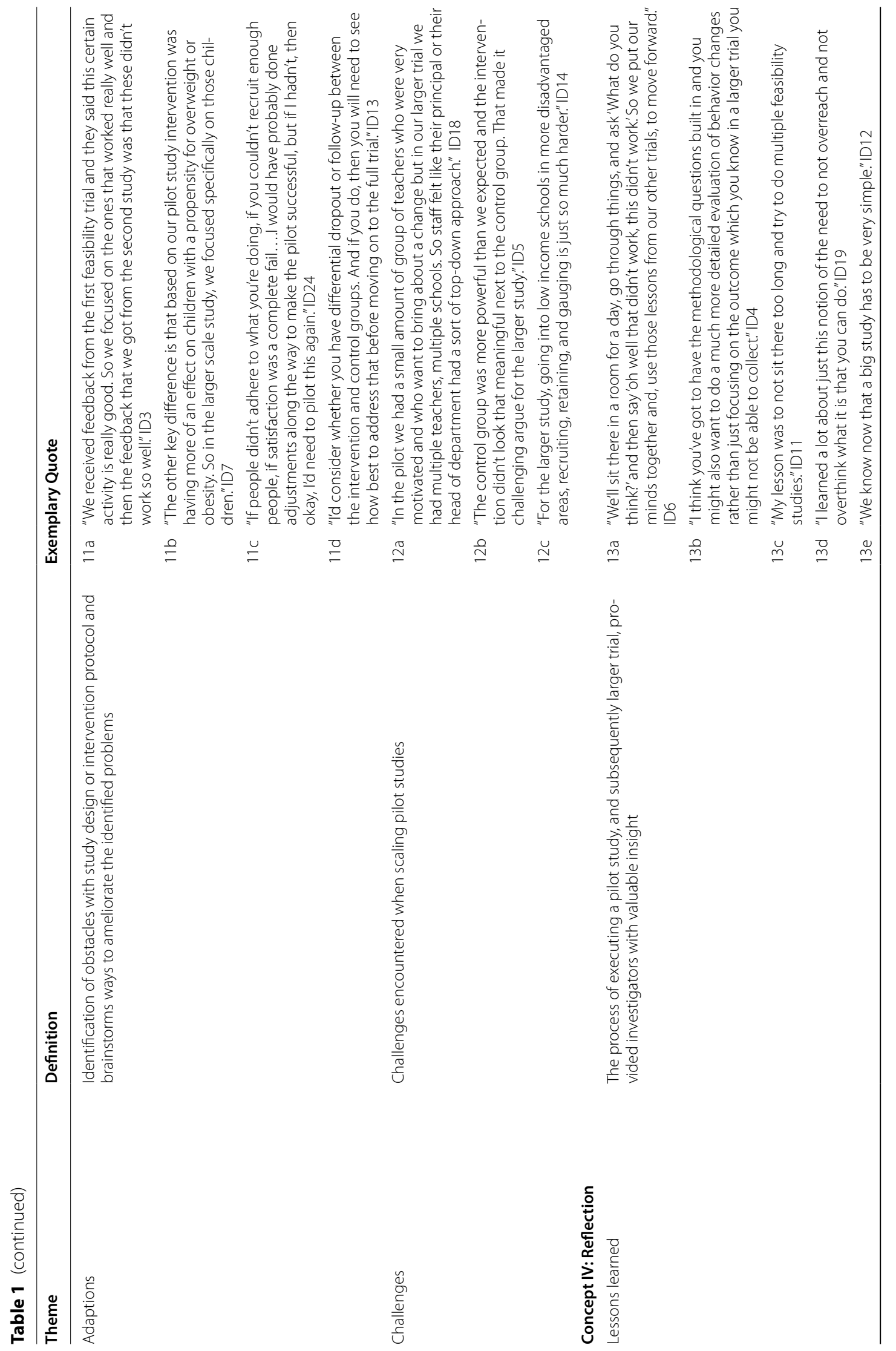




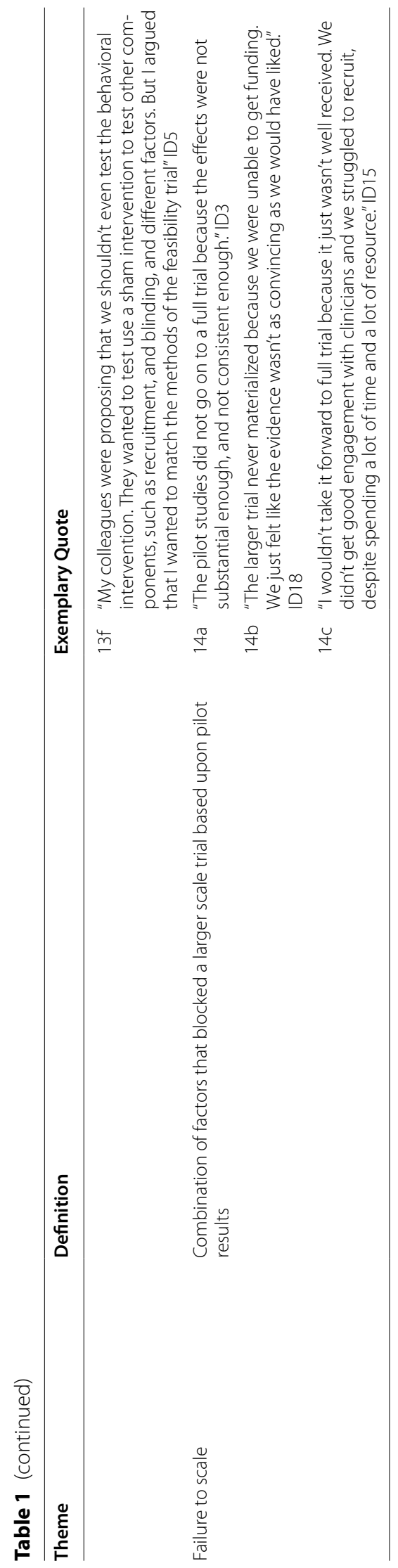


between the pilot and the larger-scale trial. This included hypothesized mechanisms of behavior change (e.g., components of behavior change theories) or intermediary behaviors related to outcomes of interest (e.g., targeting physical activity to decrease BMIz) $(n=8$; quote $1 \mathrm{~b})$ because those components were within the control of the investigator (quote $4 \mathrm{~b}$ ).

\section{Category 2: interpretation of pilot study results}

To determine if an intervention demonstrated preliminary efficacy and warranted further testing in a larger trial, interviewees discussed using multiple sources of information. These included balancing the evidence of positive results on trial-related parameters from measures of implementation and acceptability, while also considering the changes in the outcomes collected. The following components were identified as key sources of information related to interpreting the results of the pilot studies.

\section{Process implementation measures}

Interviewees described fidelity markers, such as the ease of training intervention personnel and the consistency with which manuals and protocols were delivered to participants as key markers of whether the intervention could be delivered as designed or if modifications were necessary. Measures of dose (e.g., number of sessions attended, adherence to intervention materials) and participant engagement (e.g., satisfaction, enjoyment) were also considered crucial sources of information for decision making from pilot studies (quote $5 \mathrm{~b}$ ). Investigators also sought evidence of favorable participant reception including qualitative feedback (quote $5 \mathrm{a}$ ).

\section{Indicators of preliminary efficacy}

Opinions varied on the importance and appropriate use of hypothesis testing and statistical significance when interpreting evidence of efficacy in pilot studies. When expressing their personal views, interviewees tended to view statistical testing as either inappropriate, or not a primary concern, relying instead on intuition in conjunction with the direction and magnitude of the effects along with process measures to make decisions about judging the value of an intervention and whether it should be scaled-up (quotes $6 \mathrm{a}, \mathrm{b}$ ). When commenting on perceived external expectations from grant or manuscript reviewers about inferential statistics in interpreting pilot study outcomes, authors felt expectations were confusing or impractical (quotes 6c). Setting a priori thresholds for effect sizes was mentioned by several participants $(n=5)$ though specific benchmarks were not provided. Several interviewees commented that interpreting preliminary statistical tests were prone to biased interpretation (quote 6d).

\section{Inflated effects}

Most interviewees agreed the effects from pilot studies could be inflated. Proposed reasons for inflation included recruiting motivated samples, using highly trained delivery agents, not having a control group, and increased researcher control over all aspects of the intervention delivery (quote 7a). Only nine interventionists provided potential solutions. Solutions to address inflated effects focused on designing pilot studies to more closely resemble the conditions under which the larger-scale trial would be conducted. For example, if the classroom teachers will be the delivery agents in the larger-scale trial, then the pilot should also have the classroom teachers deliver it (quote 7b, c).

\section{Category 3: scaling-up piloted interventions}

Once pilot testing was complete, interventions were often implemented on a larger scale. While some elements of the pilot interventions and trial parameters were maintained in the next phase of research, many were adapted. It was not uncommon for investigators to report changing the duration, typically from a shorter pilot (e.g., 12 weeks) to longer timeframe in the larger-scale trial (e.g., 6 months) or reducing the intensity of the intervention in the larger-scale trial from what was provided in the pilot. Notably, some investigators indicated using pilot studies to evaluate the logistics of deploying and collecting measures, though a challenge repeatedly mentioned when discussing larger-scale trials was the ability to collect measures with a substantially larger number of participants.

\section{Re-piloting interventions}

Investigators were asked to describe, either from their own experience or hypothetically, circumstances where re-piloting an intervention would be necessary based upon the results of a pilot study. Some investigators expressed rarely or never re-piloting studies $(n=3)$, while others $(n=5)$ mentioned re-piloting interventions in hopes of achieving stronger effects on their specified outcomes (quote 8a). Complete lack of engagement, inability to recruit participants, and substantial changes to intervention content were noted as signs that re-piloting was not worthwhile (quote $8 \mathrm{~b}, \mathrm{c}$ ).

\section{Strategies for scaling-up}

Investigators described strategies that helped take their pilot study to larger-scale trials. These included having strong collaborative relationships with community partners (quote 9a), allowing for more flexibility during intervention implementation (quote $9 \mathrm{~b}$ ) as well as adapting the dissemination model for an increased number of participants or delivery agents (quote 9c). 


\section{Funding of larger-scale trials}

Funding played an essential role in the execution of both pilot studies and subsequent larger-scale studies $(n=18)$. Funding was described as necessary for conducting to a pilot study, with outcomes from pilot studies deemed critical to securing additional funds to conduct a largerscale trial of the same or similar intervention (quote 10b). Thus, pilot studies were consistently viewed as a necessary prerequisite to obtaining funding for larger-scale trials. Some interviewees believed that publishing pilot studies increased their odds of successfully obtaining large-scale funding $(n=9)$. Conversely, an equal number believed that publishing pilot studies was a difficult and disincentivized process, because pilot studies were unlikely to produce significant effects or were considered lower quality studies (quote 10d, e). Other strategies for successfully obtaining funding included conducting only pilots perceived to have a greater likelihood of success at obtaining larger-scale funding (10c) and choosing primary outcomes in pilot studies likely to indicate positive change (change quote 10a).

\section{Adaptations}

Intervention components and study designs often differed between the pilot and larger-scale trials $(n=17)$. Pilot study outcomes were mentioned as useful in the identification of areas for improvement when taking the intervention to scale. Notably, investigators mentioned changing intervention intensity, duration, and study design (e.g., including randomization). These changes were driven by participant feedback in the pilot studies, funding priorities, methodological requirements of larger-scale trials, or the desire to increase efficacy of the intervention (quotes 11a-11c). Insufficient recruitment, retention, or participant satisfaction (quotes $5 \mathrm{a}, 5 \mathrm{~b}$ ) were indicative of intervention adaptations, in addition to addressing practical limitations of space (e.g., school classrooms), personnel (e.g., classroom teacher aides), intervention protocol, and material (quotes $5 \mathrm{c}, 5 \mathrm{~d}$ ) when delivering the intervention to a larger audience.

\section{Challenges}

Most investigators encountered challenges when scaling-up their pilot studies $(n=18)$. Common challenges encountered included decreased stakeholder engagement, changes in delivery personnel, and logistical difficulties collecting outcome measures (quotes 12a-12c) in a substantially larger sample of participants. According to investigators, this resulted in less meaningful results, logistical difficulties that impeded data collection, less fidelity in intervention delivery due to a change in intervention delivery agents, ultimately rendering the impact of the scaled intervention smaller than originally anticipated.

\section{Category 4: reflection}

All interviewees reflected upon their experiences piloting behavioral interventions and the adaptations or repiloting they employed. Pilot studies deemed successful were often adapted during scale-up while less successful pilot studies were re-piloted or abandoned. Several investigators reflected favorably upon applying adaptations to larger-scale studies, considering them necessary and advantageous. Others felt adaptations were errors or that re-piloting could have identified appropriate intervention components to test in a larger-scale trial, leading to more impactful interventions.

\section{Lessons learned}

The experience of scaling pilot interventions provided investigators lessons to improve future interventions (quote 13a). Several investigators mentioned including more process implementation measures in subsequent studies (quote 13b) while others prefer more pre-development or iterative pilot work (quote 13c). Designing interventions to be simple, with fewer moving parts and complexities, were mentioned as key takeaways (quote $13 \mathrm{~d}$, e). Several $(n=10)$ investigators highlighted the role of collaborating with colleagues in developing interventions, though they differed on the perceived benefits. Some investigators found additional contributions detracted from intervention's primary purpose (quote 14f) while others felt they had capitalized on collective brainstorming to improve the interventions approach (quote 14a).

\section{Failure to scale-up}

When asked to talk about experiences deploying pilot studies that had not been successfully scaled, investigators focused on a combination of multiple factors rather than singular events or influence, citing lack of sufficient effects, low participant reception, shifting funding priorities, limited researcher capacity and lack of large-scale funding for a given topic (quote 14a-14c).

\section{Discussion/conclusion}

To better understand the process of scaling-up earlystage trials, we conducted interviews with 24 investigators who had a published pilot study and subsequent trial on topics related to childhood obesity. Investigators reported that pilot studies were used to preemptively address challenges such as engaging key stakeholders, establishing trial parameters and measurement logistics, 
nonetheless, these same elements were identified as reasons scaled-up trials were less successful. These findings suggest that, while pilot studies may result in favorable trial-related parameters and evidence of preliminary efficacy, this evidence may not accurately reflect the conditions encountered during the implementation and evaluation of the subsequent scaled-up intervention.

The current study builds on previous findings indicating some components of early-stage studies have a high potential to produce exaggerated or inflated effects which diminish during subsequent, scaled-up iterations $[12,14]$. Components which were previously identified through quantitative meta-epidemiological methodology [12], such as delivery agent bias and target audience bias, were confirmed qualitatively by our participants. Major causes of inflated effects suggested by investigators included high levels of oversight in pilot studies (e.g., researcher support) and utilizing biased or highly motivated samples. Given the evident alignment between investigator-reported elements and patterns identified in the meta-epidemiologic study, the data suggests that investigators can choose to minimize or eliminate inflated effects when executing pilot studies. In doing so, the results of pilot studies could provide better evidence upon which decisions about scaling or re-piloting can be made.

Findings from this study suggest that potential conflict may be present between the need to obtain funding and the need to assess key uncertainties when piloting interventions. Investigators expressed that obtaining funding for a larger intervention was a key definition of success. To attain funding, investigators may introduce artifacts within their pilot studies that lead to inflated effects and it is these inflated effects that may be viewed more favorably from a funding perspective. Minimizing potentially inflated effects obtained from pilot studies was not mentioned as a key consideration when executing earlystage work by the investigators even though they recognized inflated effects often occur. Early-stage studies that minimize artifacts associated with inflated effects, such as ensuring the sample in the pilot study matches the sample in the larger-scale trial, have a smaller reduction in their effectiveness when scaled and a greater likelihood of demonstrating statistically significant effects in the larger-scale trial $[12,14]$. With funding as a key marker of success, investigators may knowingly or unknowingly design and execute their pilot study to ensure this success, rather than designing/executing the pilot study to understand whether or not the intervention can be adequately scaled while retaining some of its initial effectiveness.

The conflict between piloting for key uncertainties and the need to obtain funding may also be exasperated by the common two-step process for intervention development-initial piloting with small sample sizes followed by a larger-scale trial with a substantially larger number of participants. This may inadvertently force investigators to choose between fostering evidence of effects over addressing key uncertainties related to feasibility and acceptability. In other words, investigators may only have a single opportunity to establish the "value" of the intervention being piloted. Funding structures may add to this conflict by offering few mechanisms that support a multiphased developmental approach for early-stage studies which offer the opportunity to pilot and refine an intervention in progressively larger sample sizes, as opposed to conducting a single small study and using this as the only basis for which to judge the readiness of an intervention to be tested on a much larger-scale $[14,30]$. Thus, the interventionists in this study may be expressing their opinions of success as a reflection of how current funding structures are established rather than whether the process of an initial pilot followed by a larger-scale trial is the most optimal way to establish evidence for scaling.

Existing models for scaling behavioral research describe an iterative sequencing of formative work which may help address this concern, though they vary in the number and purpose of each specified step or phase of the process [18]. Similarly, our interviewees describe using a diverse sequence and size of formative studies to inform later larger-scale trials. Some investigators considered qualitative studies a "first step" while others began with smaller interventions or measurement studies. Some would not initiate pilot studies without initial confidence in the efficacy of the intervention. Others expressed a preference for "tinkering" along the way, using multiple smaller, unpublished intervention studies prior to more formal pilot work. This lack of consensus on sequencing formative work may contribute to some of the variability in pilot study utility. Clarifying the role of formative qualitative work and smaller scale "prepilot" studies in the development of later, more formal pilot studies may assist researchers in standardizing and streamlining procedures and protocols, and therein, codifying the delivered intervention and stabilizing observed effects [22]. This process can be linear, though it can also be recursive with investigators piloting interventions several times (i.e., re-piloting) when initial effects were insufficient or recruitment and retention were poor.

Investigators who perceived the results of their pilot studies to be lacking, often re-piloted or abandoned the next stage of their intervention development. However, those who felt their pilot results were promising often adapted elements of their interventions in their larger trial, sometimes involving a major reworking of the intervention. These reworkings included changing the 
intervention protocol and/or utilizing different locations, settings, or delivery agents. Investigators often perceived the process of interpreting pilot-produced evidence as nebulous, involving both quantitative markers of preliminary efficacy and process measures such as acceptability collected via mixed-methodology. This perspective of interpreting feasibility markers alongside preliminary signals of efficacy is reflective of changes in the field of behavioral research where investigators suggest testing for statistical effects in pilot studies is no longer recommended [31, 32].

There a several imitations in the current study. First, we purposively sampled only individuals who had conducted and published pilot research of behavioral interventions and subsequent scaled-up trials of the same or similar intervention. While early-stage research is widely used to develop behavioral interventions, there are a number of larger-scale behavioral trials that do not explicitly reference early-stage research that informed the intervention. Additionally, there are a large number of published pilots that do not inform later scaled-up trials. The viewpoints of authors who did not explicitly cite pilot trials and authors who have not scaled-up pilot trials are therefore not represented. Second, response bias may be present among the interviewees. Investigators who felt more comfortable discussing their early-stage work, those who had more positive experiences executing pilot studies, or who spent more time engaging in reflection on preliminary work, may have been more likely to participate. Though the 24 participants interviewed represent $63 \%$ of the entire eligible population of PIs, the potentially unique perspectives of non-respondent investigators are not represented. Additionally, it is possible that the inclusion of authors as participants may have skewed findings (AO, DL, RJ). However, it should be noted that the conceptual/theoretical approach employed for this study allowed for the inclusion of participant-authors (AO, DL, RJ) and intentional, preplanned methodological steps were employed to ensure the trustworthiness of the data. Lastly, it was not practical to conduct interviews face-to-face as the participants were located across four continents. Thus, contextual information like body language were not collected.

In conclusion, our study suggests that careful planning during the early-stage testing of an intervention can lead to pilot studies that provide important information about whether an intervention should be scaled-up. This could be achieved by supporting behavioral interventionists in designing pilot studies that closely mimic the condition encountered at scale. Additionally, guided interpretation of pilot-produced information could assist interventionists in making prudent, informed decisions regarding the necessity and appropriateness of re-piloting their interventions prior to scaling such as transparency in the rationale and a priori progression criteria in pilot studies. High-quality pilot studies have the potential to inform effective, impactful interventions to address important public health problems. However, the design, execution and interpretation of high-quality pilot studies should not be assumed to be selfevident or intuitive but rather the result of careful planning, execution, and thoughtful interpretation.

\section{Supplementary Information}

The online version contains supplementary material available at https://doi. org/10.1186/s40814-022-00991-8.

Additional file 1. Semi-Structured Interview Guide.

Additional file 2. A Priori Themes and Emergent Themes.

\section{Acknowledgements}

The authors would like to thank the participants for providing their time and insight. Additional thanks to Hannah Wilks and Alexa Taveres for assisting with transcription verification.

\section{Authors' contributions}

Lauren von Klinggraeff - Conceptualization, Methodology, Validation, Formal analysis, Data Curation, Writing - Original Draft, Visualization; Roddrick Dugger - Validation, Formal analysis, Data Curation, Writing - Review \& Editing; Anthony D. Okely - Conceptualization, Writing - Review \& Editing; David Lubans - Conceptualization, Writing - Review \& Editing; Russell (Russ) Jago - Conceptualization, Writing - Review \& Editing; Sarah Burkart - Conceptualization, Writing - Review \& Editing, Visualization; R. Glenn Weaver - Conceptualization, Writing - Review \& Editing; Bridget Armstrong - Conceptualization, Writing - Review \& Editing; Christopher D. Pfledderer - Conceptualization, Writing - Review \& Editing, Visualization; Michael W. Beets - Conceptualization, Methodology, Investigation, Resources, Writing - Review \& Editing, Visualization, Supervision, Funding acquisition. The authors read and approved the final manuscript.

\section{Funding}

Research reported in this abstract was supported by The National Heart, Lung, and Blood Institute of the National Institutes of Health under award number R01HL 149141 (Beets), F31HL158016 (von Klinggraeff), F32HL154530 (Burkart) as well as by the Institutional Development Award (IDeA) from the National Institute of General Medical Sciences of the National Institutes of Health under award number P20GM130420 for the Research Center for Child Well-Being. The content is solely the responsibility of the authors and does not necessarily represent the official views of the National Institutes of Health. Dr. Lubans is supported by a National Health and Medical Research Council Senior Research Fellowship (APP1154507). Dr. Okely is supported by a National Health and Medical Research Council Investigator Grant (APP1176858)

\section{Availability of data and materials}

Data will be made available upon reasonable request to the corresponding author.

\section{Declarations}

Ethics approval and consent to participate

Full ethics approval was received from University of South Carolina Office of Research Compliance Institutional Review Board for Human Research (Pro00086876). Eligible participants were provided with information about the study via email and given the opportunity to ask questions. Participants provided informed consent through verbal assent. All participants were given a unique study ID to ensure anonymity.

Consent for publication

Not applicable 


\section{Competing interests}

The authors declare that they have no competing interests.

\section{Author details}

${ }^{1}$ Arnold School of Public Health, University of South Carolina, 921 Assembly Street, Room 130, Columbia, SC 29205, USA. ${ }^{2}$ School of Health and Society, University of Wollongong, Wollongong, Australia. ${ }^{3}$ Priority Research Centre for Physical Activity and Nutrition, University of Newcastle, Callaghan, Australia. ${ }^{4}$ Centre for Exercise, Nutrition \& Health Sciences, School for Policy Studies, University of Bristol, Bristol, UK

Received: 10 May 2021 Accepted: 24 January 2022

Published: 7 February 2022

\section{References}

1. McCrabb S, Lane C, Hall A, Milat A, Bauman A, Sutherland R, et al. Scalingup evidence-based obesity interventions: a systematic review assessing intervention adaptations and effectiveness and quantifying the scale-up penalty. Obes Rev. 2019;20(7):964-82.

2. Indig D, Lee K, Grunseit A, Milat A, Bauman A. Pathways for scaling up public health interventions. BMC Public Health. 2017;18(1):68.

3. Milat AJ, King L, Bauman A, Redman S. Scaling up health promotion interventions: an emerging concept in implementation science. Health Promot J Austr. 2011:22(3):238.

4. Milat AJ, King L, Bauman AE, Redman S. The concept of scalability: increasing the scale and potential adoption of health promotion interventions into policy and practice. Health Promot Int. 2013;28(3):285-98.

5. Milat AJ, Newson R, King L, Rissel C, Wolfenden L, Bauman A, et al. A guide to scaling up population health interventions. Public Health Res Pract. 2016;26(1):e2611604.

6. Craig P, Dieppe P, Macintyre S, Michie S, Nazareth I, Petticrew M. Developing and evaluating complex interventions: the new Medical Research Council guidance. Brit Med J. 2008:337(7676).

7. Neta G, Sanchez MA, Chambers DA, Phillips SM, Leyva B, Cynkin L, et al. Implementation science in cancer prevention and control: a decade of grant funding by the National Cancer Institute and future directions. Implement Sci. 2015;10:4

8. Eccles MP, Armstrong D, Baker R, Cleary K, Davies H, Davies S, et al. An implementation research agenda. Implementation Science. 2009;4(1):18.

9. Peters DH, Tran NT, Adam T. In: Research AfHPaS, editor. Implementation Research in Health: A Practical Guide; 2013.

10. Anaya H, Browning M, Damschroder L, Damush T, Delevan D, Elnitsky C, et al. Quality Enhancement Research Initiative - Implmentation Guide. In: Veterans Health Adminstration [VHA], editor.

11. Wolfenden L, Ziersch A, Robinson P, Lowe J, Wiggers J. Reducing research waste and improving research impact. Aust N Z J of Public Health. 2015;39(4):303-4

12. Beets MW, Weaver RG, loannidis JPA, Geraci M, Brazendale K, Decker L, et al. Identification and evaluation of risk of generalizability biases in pilot versus efficacy/effectiveness trials: a systematic review and meta-analysis. Int J Behav Nutr Phys Act. 2020;17(1):19.

13. Chambers DA, Glasgow RE, Stange KC. The dynamic sustainability framework: addressing the paradox of sustainment amid ongoing change. Implement Sci. 2013;8:117.

14. Beets MW, von Klinggraeff $L$, Burkart $S$, Jones A, loannidis JPA, Weaver RG, et al. Impact of risk of generalizability biases in adult obesity interventions: A meta-epidemiological review and meta-analysis. Obes Rev. 2021;n/a(n/a):e13369.

15. Eldridge SM, Lancaster GA, Campbell MJ, Thabane L, Hopewell S, Coleman $\mathrm{CL}$, et al. Defining feasibility and pilot studies in preparation for randomised controlled trials: development of a conceptual framework. Plos One. 2016;11(3):e0150205.

16. Orsmond $\mathrm{Gl}$, Cohn ES. The Distinctive Features of a Feasibility Study: Objectives and Guiding Questions. OTJR (Thorofare N J). 2015;35(3):169-77.

17. Pearson N, Naylor P-J, Ashe MC, Fernandez M, Yoong SL, Wolfenden L. Guidance for conducting feasibility and pilot studies for implementation trials. Pilot Feasibility Stud. 2020;6(1):167.
18. Czajkowski SM, Powell LH, Adler N, Naar-King S, Reynolds KD, Hunter CM, et al. From ideas to efficacy: The ORBIT model for developing behavioral treatments for chronic diseases. Health Psychol. 2015;34(10):971-82.

19. Freedland KE, King AC, Ambrosius WT, Mayo-Wilson E, Mohr DC, Czajkowski SM, et al. The selection of comparators for randomized controlled trials of health-related behavioral interventions: recommendations of an NIH expert panel. J Clin Epidemiol. 2019:110:74-81.

20. Levati S, Campbell P, Frost R, Dougall N, Wells M, Donaldson C, et al. Optimisation of complex health interventions prior to a randomised controlled trial: a scoping review of strategies used. Pilot Feasibility Stud. 2016;2(1):17.

21. Bowen DJ, Kreuter M, Spring B, Cofta-Woerpel L, Linnan L, Weiner D, et al. How we design feasibility studies. Am J Prev Med. 2009;36(5):452-7.

22. Onken LS, Carroll KM, Shoham V, Cuthbert BN, Riddle M. Reenvisioning clinical science: unifying the discipline to improve the public health. Clin Psychol Sci. 2014;2(1):22-34.

23. Eldridge SM, Chan CL, Campbell MJ, Bond CM, Hopewell S, Thabane $L$, et al. CONSORT 2010 statement: extension to randomised pilot and feasibility trials. BMJ. 2016;355:15239.

24. Ehrhart MG, Aarons GA, Farahnak LR. Assessing the organizational context for EBP implementation: the development and validity testing of the Implementation Climate Scale (ICS). Implementation Sci. 2014;9(1):157.

25. World Medical Association. World Medical Association Declaration of Helsinki. Ethical principles for medical research involving human subjects. Bull World Health Organ. 2001;79(4):373.

26. Robinson WS. The logical structure of analytic induction. Am Sociol Rev. 1951;16(6):812-8

27. Bogdan R, Biklen SK. Qualitative research for education: Allyn \& Bacon Boston, MA; 1997.

28. Gale NK, Heath G, Cameron E, Rashid S, Redwood S. Using the framework method for the analysis of qualitative data in multi-disciplinary health research. BMC Med Res Methodol. 2013;13(1):117.

29. Shenton AK. Strategies for ensuring trustworthiness in qualitative research projects. Education for Information. 2004;22:63-75.

30. Beets MW, von Klinggraeff L, Weaver RG, Armstrong B, Burkart S. Small studies, big decisions: the role of pilot/feasibility studies in incremental science and premature scale-up of behavioral interventions. Pilot Feasibility Stud. 2021;7(1):173.

31. Sim J. Should treatment effects be estimated in pilot and feasibility studies? Pilot Feasibility Stud. 2019:5(1):107.

32. Leon AC, Davis LL, Kraemer HC. The role and interpretation of pilot studies in clinical research. J Psychiatr Res. 2011;45(5):626-9.

\section{Publisher's Note}

Springer Nature remains neutral with regard to jurisdictional claims in published maps and institutional affiliations. 